\title{
«Das Wesentliche ist für die Augen unsichtbar»
}

\author{
Literarische Sehhilfen in Antoine de Saint-Exupérys Petit Prince
}

Franzisca Pilgram-Frühauf

Man stelle sich vor: Ein kleines Kind streckt einem erwachsenen Gegenüber erwartungsvoll und mit leuchtenden Augen eine soeben angefertigte Zeichnung entgegen. Die Bezugsperson wirft einen Blick darauf: Erkennt sie spontan, was dargestellt ist, so benennt und lobt sie das Bild; denn vielleicht weiss sie, dass viel davon abhängt, ob solche ersten Werke mit Verständnis und Respekt aufgenommen oder verächtlich als blosses Gekritzel beiseite gelegt werden. Was aber, wenn sie aus den Farbklecksen und wirren Strichen nicht auf Anhieb erschliessen kann, was das Kind zeigen möchte? Ist dann anzunehmen, dass die kindlichen Augen mehr sehen als die erfahrenen und geschulten Augen der Erwachsenen? Sehen sie womöglich etwas, das hinter den sichtbaren Farben und Formen verborgen ist?

Diesem hermeneutischen Problem möchte ich im Folgenden auf die Spur kommen. Nach ein paar Vorüberlegungen soll untersucht werden, wie die Herausforderung des Unsichtbaren in SaintExupérys Erzählung vom kleinen Prinzen verarbeitet wird.

\section{Repräsentation vs. Präsentation}

Auch ohne die frühkindliche Entwicklung eingehend studiert zu haben, scheint bereits klar: Man wird dem Problem kaum gerecht, wenn man die Diskrepanz zwischen dem, was das Kind zeichnen will, und dem, was der Erwachsene auf dem Papier tatsächlich sieht, auf ein Defizit des kindlichen Blicks oder auf eine mangelhafte zeichnerische Begabung zurückführt. Wie der Kinderarzt und -psychiater Heinz Stefan Herzka bestätigt, gründen die Schwierigkeiten vielmehr darin, dass das Kind eine grundsätzlich andere Sichtweise hat als der Erwachsene: «Bis zum Schulalter und noch darüber hinaus zeichnet es von innen heraus, was seine Gefühle bewegt, was seine Gedanken erfüllt. Es zeichnet die Welt, wie es sie erlebt, und nicht, wie sie der Erwachsene sieht.» ${ }^{1}$ Die erlebnisorientierte

1 H.S. Herzka, Das Kind von der Geburt bis zur Schule. Bilderatlas und Texte zur Entwicklung des Kindes, Basel 1972, 101. 
Ausprägung des bildnerischen Gestaltens sei charakteristisch für das so genannte «Stadium des unschematischen Zeichnens», das bis ins siebte Lebensjahr andauern könne: «Das Kind bildet dabei nicht die äussere Umwelt ab, indem es sie nachzuzeichnen oder nachzumalen versucht, sondern es bringt innere Bilder zu Papier, die von dem bestimmt sind, was im Fühlen und Denken des Kindes Gesicht hat. [...] Grössenverhältnisse entsprechen nicht der Wirklichkeit, oft aber der Bedeutung, welche bestimmte Dinge für das Kind haben.»²

Dem markanten Unterschied, den Herzka zwischen den Bildkonzepten des Kindes und des Erwachsenen feststellt, hat sich aus einer völlig anderen Warte auch der Kunsthistoriker Georges Didi-Huberman gewidmet. In seinen umfangreichen Studien versucht er so «vor einem Bild» zu stehen, dass er nicht nur - wie bei Kunstexperten sonst üblich - nach dem bildhaft Dargestellten (der sog. Repräsentation) fragt, die Bestände ikonographischen Wissens abruft und begriffliche Erkenntnis anstrebt, sondern auch für die ereignishafte Präsentation des Visuellen sensibel wird ${ }^{3}$. Mit der neuen Betrachtungsweise verschwindet bis anhin Sichtbares aus dem Blick, Unsichtbares wird entdeckt; denn das Bild ist nur noch vage auf ein Dargestelltes zurückbezogen, präsentiert vielmehr - vielleicht auch nur betrachterspezifisch - einen Vorgang, der seinen Ursprung in etwas Verborgenem hat.

Saint-Exupérys Kleiner Prinz ${ }^{4}$ öffnet schliesslich noch einen dritten Zugang zu den beiden Bildkonzepten, die hier mit der Repräsentation bzw. der Sichtweise des erwachsenen Auges und der Präsentation bzw. der Sichtweise des kindlichen Auges in Verbindung gebracht werden: Der literarische Text, der insgesamt von den Möglichkeiten der Einbildungskraft handelt, und die 46 Aquarelle des Autors durchdringen sich auf vielschichtige Weise. Die Bilder haben nicht nur die Funktion, die Geschichte darstellend zu illustrieren, sondern bilden oft auch den Rahmen, in den sich der Text graphisch einfügt (vgl. 24, 41). Und, was im Folgenden besonders zur Geltung kommen soll: Die Schlüsselstellen des Werks nehmen auf Bilder Bezug und thematisieren das Abenteuer des Sehens von Unsichtbarem.

\footnotetext{
Ebd., 105.

G. Didi-Huberman, Vor einem Bild, aus dem Franz. von R. Werner, München/ Wien 2000, S. 24f. [orig.: Devant l'image. Question posée aux fins d'une histoire de l'art, Paris 1990].

4 A. de Saint-Exupéry, Der Kleine Prinz. Mit Illustrationen des Autors, Zürich/ Hamburg 2006, [orig.: Le Petit Prince, Paris 1946].
} 


\section{Ungeheure Urwaldtiere}

Der auktoriale Erzähler erinnert sich einleitend an ein Bild, welches er als Sechsjähriger in einem Buch über den Urwald entdeckt hat und das einen gewaltigen Eindruck auf ihn hinterliess. Es stellt eine Riesenschlange dar, die gerade dabei ist, eine Beute zu verschlingen - «ohne sie zu zerbeissen», wie der Kommentar lakonisch zu verstehen gibt. Als Leser/Leserin des Kleinen Prinzen bekommt man eine «Kopie»" dieses Bildes zu sehen. Gemäss der Terminologie Didi-Hubermans handelt es sich um die Repräsentation einer Repräsentation; denn genau genommen ist das Original nicht in der naturkundlichen Abbildung, sondern in vivo, im gefahrvollen Dschungel selbst zu suchen.

Die nächste Illustration zeigt, wie das Kind das Bild aus dem Buch verarbeitet, gewissermassen «verdaut» hat: Sie nimmt das Motiv auf und variiert es. Im Bauch der Reisenschlange befindet sich nun ein Elefant, der, bereits verschlungen, die schlangenhaften Umrisse ins Unkenntliche verzerrt. Die Augen sämtlicher "grossen Leute», denen der Knabe sein «Meisterwerk»" vorführte, waren der Präsentation gegenüber verschlossen, glaubten anhand der Konturen einen Hut zu erkennen und betrachteten das Bild als misslungen. Sie verstanden es nur mit der Sehhilfe von Erklärungen, d.h. in diesem Fall mit einer weiteren Zeichnung, die das Innere der Boa sichtbar machte.

Inwiefern geht es in dieser Vorgeschichte zur Haupthandlung des Kleinen Prinzen um Unsichtbares? Im Zentrum steht die Illustration, die den Elefanten dem Blick des Bildbetrachters entzieht. Nur schemenhaft weist die groteske Form seiner Umhüllung auf ihn hin. Die Unsichtbarkeit des grossen Tieres führte dazu, dass aus der Zeichnung ein «Misserfolg» wurde, so dass der junge Maler beschloss, von einer «grossartigen Laufbahn» als Künstler abzusehen und einen solideren Beruf zu wählen. ${ }^{7}$ Das Kind konnte die Schlange zwar im Längsschnitt nachzeichnen und den unsichtbaren Verdauungsvorgang aufdecken, aber es empfand es als allzu ermüdend, in Rücksicht auf die vernünftigen Deutungen der Erwachsenen sein Bild wieder und wieder erklären zu müssen. Was es zum Zeichnen veranlasst hatte, konnte es den «grossen Leuten» bis zum Schluss nicht verständlich machen. Die Eindrücke der Urwalddarstellung, das Fremde, Beängstigende oder Faszinierende daran, blieben unsichtbar und unerklärt. Sie spiegeln sich lediglich in der Frage, die das Kind stellte, während es den

\footnotetext{
5 Ebd., 7.

6 Ebd.

7 Ebd., 8.
} 
Erwachsenen sein Bild zeigte: ob ihnen die Zeichnung nicht Angst mache. ${ }^{8}$ Die Betrachter nahmen das Präsentierte nicht wahr und überhörten die Frage, denn das im Bild Verborgene vermochte bei ihnen keine Wirkung zu entfalten.

\section{Schachtel mit Luftlöchern}

Nicht nur mit den Erwachsenen und dem Leser/der Leserin des Buches kommt der Ich-Erzähler über Bilder in Kontakt: Als er viele Jahre nach seinen desillusionierenden Kindheitserfahrungen nach einer Notlandung in der Wüste, zwischen Leben und Tod schwebend, ${ }^{9}$ dem kleinen Prinzen begegnete, entwickelte sich die Freundschaft über ein Bild: Kurz nachdem das «kleine, höchst ungewöhnliche Männchen» überraschend aufgetaucht war, bat es den fremden Mann, ihm ein Schaf zu malen. Das einzige, was der gestrandete Pilot von früher her noch zeichnen konnte, war eine "geschlossene Riesenschlange»" ${ }^{10}$ - ein Bild, das der Auftraggeber auf Anhieb verstand. Aber weder damit noch mit den gut gemeinten Versuchen, doch noch ein Schaf darzustellen, gab sich der kleine Prinz zufrieden, denn sie blieben allesamt hinter seinen Ansprüchen zurück: Das erste Schaf war zu kränklich, ein anderes trug fälschlicherweise Hörner und ein drittes sah alt und gebrechlich aus. ${ }^{11}$

Der Ich-Erzähler kam zum Schluss, dass das Verlangte anscheinend nicht darstellbar war - wenigstens nicht mit seinen bescheidenen Zeichenkünsten -, und sah nur noch einen Ausweg: es als Unsichtbares vorzuführen. Gezeichnet wurde sodann eine Schachtel mit Luftlöchern, vom Erzähler als Behausung des vorgestellten Ideal-Schafs interpretiert. Dieses Bild wurde vom kleinen Prinzen widerspruchslos akzeptiert.

Während die elastische Schlange die Umrisse des Elefanten andeutungsweise sichtbar werden lässt, hat die gezeichnete Schachtel, die ein lebendiges Schaf verbirgt, mit diesem keine Gemeinsamkeit im Sinne eines Abbildungsverhältnisses. Sie enthält allerdings Luftlöcher, damit der Inhalt atmen kann! Es handelt sich um Zeichen, die zum Appell werden, das Unsichtbare zu imaginieren, so dass es präsent wird.

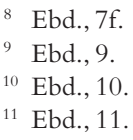




\section{Leere Wüstenlandschaft}

Die letzte Illustration des Buches zeigt eine leere Wüstenlandschaft. Ein einziger leuchtender Stern markiert den Himmel. Der kleine Prinz ist verschwunden. Hier steht der vorgestellte Inhalt noch stärker im Zeichen der Unsichtbarkeit als zuvor: Er ist abwesend, nicht nur verborgen.

Bei diesem dritten und letzten Beispiel bleibt offen, ob das Unsichtbare (wieder) präsent werden kann, d.h. ob der Leser/die Leserin die Geduld aufbringt, in der Einöde auf den kleinen Prinzen zu warten, wozu ihn der Erzähler auffordert. Angespornt durch den begleitenden Text, rekonstruiert die Phantasie möglicherweise nur das vorletzte Bild des Buches, auf dem der Prinz in derselben Landschaft eben noch zu sehen war. Dabei geht nicht nur vergessen, dass alle Porträts des kleinen Prinzen nur einen völlig unzulänglichen Eindruck vermitteln und keineswegs repräsentativ sind für den, den der Ich-Erzähler kennen und lieben gelernt hat. ${ }^{12}$ Vielmehr ist damit in letzter Konsequenz auch der Stellenwert des Textes in Frage gestellt, der von der Freundschaft mit dem kleinen Prinzen erzählt. Handelt es sich lediglich um eine sprachliche Fata morgana, die der Extremsituation der Wüste entspringt?

Dies führt mich abschliessend zur Frage nach der Rolle der Sprache, welche die Illustrationen umgibt. Die Antwort wird auch zum Prüfstein für die eingangs geschilderte Situation: Was sagen «grosse Leute» zu einer (Kinder-)Zeichnung, auf der weniger sichtbar ist, als man gewöhnlich erwartet?

\section{Poesie als präsentierende Sprache}

Der Ich-Erzähler scheitert zwar als (darstellender) Maler, kommt aber dank seinem Wüstenerlebnis, der geheimnisvollen Begegnung mit dem kleinen Prinzen, zu einer Geschichte und findet eine Sprache, in der man "wirklich» redet. ${ }^{13}$ Denn grundsätzlich kann man in SaintExupérys Buch beobachten:Je weniger die Zeichnungen ihren Inhalt repräsentierend wiedergeben, desto wichtiger wird die Sprache, welche die Bilder umgibt und das Unsichtbare im Sichtbaren postuliert. Im Fall der Schlange gibt zunächst eine repräsentierende Abbildung das Original wieder. Zum daran anknüpfenden eigenen Kunstwerk liefert der Erzähler all jenen, die über keinen Röntgenblick verfügen,

12 Ebd., 11.

13 Ebd., 9. 
eine klärende Zeichnung nach, die keines besonderen Kommentars mehr bedarf, zumal bereits die entscheidende Frage des Knaben, ob sein Bild nicht Angst mache, bloss auf Unverständnis stösst. Die dargestellten Schafe in der zweiten Episode sind klägliche Vorübungen, an denen man sich bei der Vorstellung des Schafes, das in der Schachtel lebt, besser nicht orientiert. Die Luftlöcher, die auf den Bewohner des behelfsmässigen Stalls hinweisen, müssen genügen. Ins Zentrum rückt aber die Interpretation, die der Erzähler dem kleinen Prinzen im Gespräch nahe legt und die auch den Leser/die Leserin dazu stimuliert, sich das Schaf in der Schachtel vorzustellen - ähnlich, wie man auch später im Niemandsland der Wüste auf das Wiedererscheinen des Prinzen hoffen soll. Bei diesem Schlussbild ist der Erzähler nun aber ganz auf die Leserinnen und Leser angewiesen, die auf die Präsentation des Unsichtbaren warten können und ihm zu schreiben gewillt sind, wenn sein Freund "wieder da ist» ${ }^{14}$. Sie setzen dann gewissermassen den Text der Geschichte fort, indem sie von ihren eigenen Erfahrungen erzählen.

Die drei Episoden führen sowohl eine graduelle Abnahme der bildhaften Repräsentation von Wirklichkeit vor als auch eine Steigerung des Unsichtbaren und eine Intensivierung des poetischen Appells, Unsichtbares halluzinatorisch wahrzunehmen. Diese Verlagerung auf die präsentierende Kraft einer "wirklichen», oder vielleicht noch besser: verwirklichenden Sprache korrespondiert mit dem, was im Text des Kleinen Prinzen als "Geheimnis» eingeführt wird: Dieses wird zunächst vom Fuchs an den Prinzen, dann von diesem an den Erzähler weitergegeben und schliesslich auch im Laufe der Lektüre beim Betrachten der Illustrationen eingeübt: «Man sieht nur mit dem Herzen gut. Das Wesentliche ist für die Augen unsichtbar.» ${ }^{15}$ Bildet diese sprachliche Formel den hermeneutischen Schlüssel zum Verständnis der Bilder von der verdauenden Schlange, der bewohnten Schachtel, dem leeren Wüstenraum, in welchem der kleine Prinz wieder erscheinen könnte, - und vielleicht auch den Schlüssel zu einem besseren Verständnis für erste Kinderzeichnungen?

Man darf mich nicht falsch verstehen: Die zwei Arten des Blicks, wie ich sie hier mit dem für Repräsentationen empfänglichen Auge der Erwachsenen und der erlebnis- und gefühlsorientierten Sichtweise der Kinder verbinde, sind keine entwicklungspsychologischen Stadien oder kunsttheoretischen Paradigmen, die sich gegenseitig ausschliessen. Interpretation von Bildern ist immer - und oft unvorhersehbar - eine Mixtur von beidem. Der entscheidende

\footnotetext{
14 Ebd., 93.

15 Ebd., 72.
} 
Punkt, das «Geheimnis», mit dem man im Kleinen Prinzen konfrontiert wird, liegt vielmehr in den Übergängen zum «Sehen mit dem Herzen» und in der Frage, wodurch diese ausgelöst werden. Wenn die sichtbaren Konturen des Dargestellten verbleichen, Löcher in den Mittelpunkt der Zeichnung rücken und die Leere des Raums präsent wird, so liegt dies daran, dass die Bilder nicht mehr nur darstellen, sondern etwas Unheimliches, Unerwartetes oder Unvollständiges mitführen, das altbewährte Sehgewohnheiten herausfordert und die Betrachter zur eigenen Beteiligung zwingt - sei es dass man auf die Fragen und Interpretationen anderer Betrachter reagiert oder selbst im Bild etwas Neues entdeckt. Das Unsichtbare erschliesst so auch Räume für die Beziehung zwischen dem Kind und dem erwachsenen Betrachter, so dass eine "wirkliche», d.h. dialogische Sprache entsteht, obschon oder vielleicht gerade weil die Blickrichtungen unterschiedlich sind.

In letzter Konsequenz kann es in solchen Situationen des überraschenden Übergangs zum Unsichtbaren auch geschehen, dass die «grossen Leute» einen Blickwechsel mit sich selbst vollziehen müssen und dass ihnen Bilder erscheinen, denen etwas geheimnisvoll Verborgenes anhaftet, wie man sie aus der eigenen Kindheit kennt. Denn, wie es im Vorwort zum Kleinen Prinzen heisst, man vergesse nicht: «Alle grossen Leute sind einmal Kinder gewesen [...].» ${ }^{16}$

— Dr. des. Franzisca Pilgram-Frühauf ist Assistentin am Lehrstuhl für Systematische Theologie, insb. Hermeneutik und Fundamentaltheologie an der Universität Zürich.

${ }^{16}$ Ebd., 5. 